I performed a second operation on a woman who had had a cholecystectomy, and in that second operation I removed a handful of stones from her dilated common duct. We must not conclude, therefore, that cholecystectomy is always wrong. The point I want to make is that there is overwhelming proof that in man the gallbladder is an important organ; and that therefore, unless there is a good reason for taking it out, it should be allowed to remain in. On the other hand, I think it is as conclusively proved that in many of theses cases the gallbladder has already been put out of commission by pathologic processes. To allow that sort of gallbiadder to remain in an abdomen is to do imperfect work.

\section{OBSERVATIONS ON THE EXCRETION OF DYES *}

\author{
RUSSELL L. CECIL, M.D. \\ AND \\ RICHARD WEIL, M.D. \\ NEW YORK
}

The excretion of dyes offers a problem which is of interest from a variety of standpoints. It has a physiologic application on account of the light which it may throw on the functions of the excretory organs, and on the mechanism utilized by the body for the removal of waste material. Pharmacologically, it offers a simple method for the investigation of relationship between chemical structure and excretory function. Finally, it affords the clinician an instrument of pres cision in determining excretory activity. The method was introduced many years ago in the study of kidney function, and a variety of dyes have been employed for this purpose, of which the one in most general use today is phenolsulphonephthalein. An attempt has been made to study liver function in a similar manner, but the procedure has not proved entirely successful. The present paper reports on certain observations which throw new light on various aspects of the problem.

It has been found that some of the dyes of the diazo series are tolerated in large amount when given intravenously to human beings. We have injected in one dose as much as $1 \mathrm{gm}$. of Congo red in 2 per cent. solution without apparently disturbing in any way the general bodily equilibrium. The same quantity of trypan red has been given without injurious effect. It is of interest to note that the dye when given in such amounts circulates in the blood for several days unchanged, and may be demonstrated by the discoloration of the serum.

Dyes have been injected into human beings only after a thorough test of their toxicity in animals, the guinea-pig and the rabbit having been chiefly used for this purpose. The toxicity of the various diazo dyes varies markedly, as has been pointed out by Evans. However, there does not seem to be any direct relationship between chemical composition and toxicity. The side-chain theory of Ehrlich, which postulated a special affinity on the part of the cells for certain chemical side-chains of the molecule, is not supported by the facts. Slight variations in composition, such as an alteration in the relative positions of two sidechain groups of the naphthalene nucleus, may result in a distinct change in toxicity. The addition of a

* From the Department of Experimental Medicine, Cornell University Medical College, New York.
${ }^{*}$ Read before the Section on Pharmacology and Therapeutics at the
Sixty-Eighth Annual Session of the American Medical Association, liew York, June, 1917. single sulphonate group to two such preexisting groups in the molecule, a change of no apparent significance ( since there may be five of these groups in a nontoxic dye, such as trypan red) may result in an enormous increase in toxicity; this is true of heliotrope $2 \mathrm{~B}$ as compared with Congo violet. But at the same time the relative positions of the side-chains in the naph thalene group have been changed. Why this change should so completely alter the reaction of the body is not clear. The physiologic basis of these effects is for the most part quite obscure. It is of importance that the same dye, such as trypan blue, may be considerably more toxic for one species, such as the guinea-pig, than for another, such as the rabbit.

The tendency of the body is to rid the blood of this foreign material. A certain amount is taken up by the cells of the tissues, and these cells then undergo a marked discoloration. Such cells belong to the phagocytic group, and have been called "scavenger cells" by Evans. After intravenous injection into human beings of the Congo red or trypan red, the skin may assume a diffuse reddish color, the intensity of which varies with the amount injected. This discoloration gradually fades away. Inflammatory tissues also have a marked tendency to take up the color. In working with experimental arthritis, Cecil has found that trypan blue lodges in large amount in the tissue of infected joints, and Weil has shown that Congo red produced marked discolorations of the necrotic areas of malignant new growths in human beings. We have found that the dye is also excreted into pleural fluids, and is present in mucopurulent exudation from the respiratory tract, as in bronchitic sputum. We have found that the dyes are excreted on the surface of external ulcerated areas, including both ulcerated new growths and benign lesions, such as chronic varicose ulcers. Congo red, for example, when given in amounts of $0.5 \mathrm{gm}$. intravenously in human beings, appears on the surface of the ulcer in about an hour, and is then continuously demonstrable for several days. The dye certainly has no injurious effect on the ulcer, and has seemed at times to exercise a beneficial influence on the healing.

The excretion of dyes by external ulcers suggested the possibility that ulcers of the gastro-intestinal tract might perhaps act in similar fashion. We have therefore studied gastric ulcers from this standpoint. Congo red, as we have already reported in a short preliminary paper, was found in the stomach contents of a number of patients with gastric ulcer. In these cases it was usually present in very large amounts, so that the filter papers through which the contents were passed showed a heàvy red deposit. In a number of cases of gastric carcinoma the same observation was made. Thus the conditions seemed most favorable for the development of a differential diagnostic test for diseases of the stomach. On the other hand, there were some cases both of ulcer and of cancer which failed to give a reaction, while there were also occasional control cases in which neither ulcer nor cancer was present in which the stomach contents showed a small amount of dye.

In trying to account for the latter group, we found that Congo red is excreted in large amounts in the bile, and that the reflux of bile into the stomach may present a serious complication in the utilization of the method as a gastric test. This fact necessitated the study of dyes in relation to their excretion by the liver. Congo red is the only dye of which the excretion in 
human bile has been directly investigated. A patient with a postoperative biliary fistula, but without any evidence of inflammatory conditions in the biliary tract or liver, received $0.5 \mathrm{gm}$. of the Congo red intravenously. Within one-half hour the dye appeared in the bile and continued to be excreted by that route for more than twelve hours. If dyes were to be used for the diagnosis of gastric conditions, it appeared to us to be essential to utilize such dyes as were not excreted by the bile, in order that this source of error might be excluded. The method which we employed to determine whether or not dyes were excreted by the liver was to give an animal an intravenous injection, and then after two or three hours to aspirate the contents of the gallbladder. This operation can be carried out on guinea-pigs under ether. The laparotomy wound heals well, and the animals ordinarily recover and become available for a second experiment. The discoloration of the bile offers immediate evidence of the presence of the dye. At times the color of the dye in the bile is somewhat different from that of the original watery solution. Thus Bordeaux COV may have a pinkish instead of a lilac color. Ordinarily, the addition of a few drops of peroxid alters this to the usual color, thus showing that partial reduction is responsible for the change.

It was found that dyes could be divided into three classes with reference to their excretion by the liver. These classes, of course, include only such dyes as were not toxic and could therefore be injected intravenously. In the first group are the dyes which are excreted by the liver and are present in the bile. Among such dyes, in addition to Congo red, may be mentioned neutral red, safranin, methylene blue, Biebrich scarlet, benzo-azurin, and oxamin violet. The second group comprises the dyes which do not appear in the bile. Such are rosanilin, pyronin, trypan red, orange $G$, eosin, indigocarmin, azur, and a number of others. The third group comprises those dyes which make their appearance in the bile occasionally, but not regularly, when injected in the routine amount of 0.5 c.c. of a 1 per cent. solution; examples of this group are Bordeaux COV, Congo rubin, and azoblue.

The chemical or physical basis of these differences is entirely obscure. Congo Corinth differs from Congo red only in the substitution of an $\mathrm{OH}$ for an $\mathrm{NH}$ group, yet the former dye is not excreted by the bile, while the latter is.

A series of observations have been made on the excretion of these dyes not only by the liver of normal animals, but of such animals as have received injections of phosphorized oil. In the latter the liver undergoes marked fatty degeneration, and whether the animal dies or recovers depends on the dose. In such poisoned animals the dyes of the first group are excreted in the bile exactly as in normal animals. Those dyes belonging to the second group, which are never excreted by the normal liver, are never excreted by these diseased livers. The dyes of the third group, toward which the normal liver exhibits a certain latitude, are always excreted by the diseased organ. These observations indicate the likelihood of finding some dye, the presence of which in the bile will always indicate a diseased process in the liver; as yet, however, this object has not been accomplished.

For the diagnosis of gastric disease, we have tested some of the dyes in group two, which are never present in the bile. The dye which has been most thoroughly studied in this connection is trypan red. This dye has certain obvious advantages. It is very soluble, is nontoxic on intravenous injection, is excreted promptly and profusely on the surface of external ulcerations, and does not appear in the bile. These characteristics seem obviously to determine its availability for the purpose. It has been employed in a series of nine cases of organic stomach disease, including ulcer of the duodenum, and ulcer or cancer of the stomach, in which the diagnosis was established with certainty, either by operation or the Roentgen ray. In not a single one of these cases has the trypan red ever been found in gastric contents. Here, then, is an absolute difference in the behavior of the dye in skin ulcers and in gastro-intestinal ulcers. It seems difficult to account for this difference on the basis of any. differences in the nature of the two types of ulceration, and we are therefore inclined to the view that chemical conditions might be such as to obscure the presence of the dye, even if actually excreted in the stomach. Experiment has indicated that this possibility does, indeed, exist. Dilute hydrochloric acid somewhat decolorizes solutions of trypan red when left in contact therewith. If hydrogen peroxid is added to the mixture, decolorization is complete and much more rapid, yet hydrogen pefoxid alone produces hardly any alteration. If we assume that oxidases are present in all the tissues, especially in granulating surfaces, the difference in the coloration between gastric and cutaneous ulcers might conceivably be explained on this ground.

Pyronin has been tried out in cases of gastric ulcer, but also without result.

Experimentation is now under way in the excretion of these dyes by experimental gastric ulcers in dogs. and it is hoped that this method will throw some light on the problematic conditions which we have encountered in human beings.

We desire again to draw attention to the different manner in which the liver behaves toward dyes, excreting some and not others, and to the fact that this function can be modified by disease of the organ; furthermore, to the fact that ulceration of the gastro-intestinal tract appears to act differently as regards the excretion of dyes from ulceration of the skin surface.

123 East Sixty-Second Street-970 Park Avenue.

\section{ABSTRACT OF DISCUSSION}

Dr. Arthur D. Hirschfelder, Minneapolis: This work represents a most ingenious application of a phenomenon which in itself is interesting. A few years ago Dr. M. C. Winternitz and $I$ began a series of experiments on pneumonia in rabbits and found, just as the essayists have found, that trypan blue and trypan red went almost specifically into the diseased tissues, so that they marked off disoused areas of the lung and would also go into the fibrinous exudate of a pleuritis or a pericardial effusion. These dyes were absorbed by fibrin, but entered everywhere into diseased tissue. Similar observations were made by Camp and Bell in Minneapolis for other local infections, and we also have been in the habit of using injections of trypan blue to mark off burned areas or areas otherwise injured. I think it represents as sure a method as one could desire for the detection of necrotic areas, but the applications that Drs. Weil and Cecil have made-to use this for the excretion through ulcerated surfaces-is extremely interesting and, I hope, practical. One point, which probably has also occurred in the experience of Drs. Cecil and Weil, which struck us in working with trypan blue in general is the variability of the toxicity of the benzidin dyes based on their colloidal state. If we allow the trypan blue to stand for a short time the dispersion or suspension of the dye is apparently changed. The ordinary suspension or solu- 
tion of trypan blue represents a molecular weight which is about four times the theoretic molecular weight. If allowed to stand, definite visible aggregates occur. The longer it stands the greater the toxicity. This is important in the practical application of the dyes of the benzidin group. They must be used with distilled water and very fresh solutions must be used or toxicity will result.

Dr. Robert A. Hatcher, New York: Dr. Weil informed us that the liver of the dog poisoned with phosphorus does not behave toward certain dyes as does the liver of the normal dog; hence we investigated the capacity of such poisoned livers to remove strychnin from the circulation and destroy it. Dogs were given phosphorus and when moribund the liver was excised and perfused with a solution of strychnin. The results were practically identical with those obtained with normal livers.

Dr. Fred I. Lackenbach, San Francisco: Another interesting field of investigation is the use of these dyes as therapeutic agents. There has been some work done with fuchsin in the treatment of tuberculosis of the bladder and kidney and in gonococcus infections. Dr. Vecki published a paper about a year ago on the employment of fuchsin solution in strengths of 0.25 to 1 per cent. for irrigating the bladder and urethra. It is important to use the solutions as fresh as possible and fuchsin of high purity; it is otherwise irritating. While the literature on the subject is not large, it is interesting and should stimulate investigation.

Dr. Otto Rauben heimer, Brooklyn : From a historic standpoint it is interesting that the "pigmentarii," or color makers, of old Rome have been revived in our present age. In regard to the excretion of dyes I would say that in 1910 a number of samples of urine were brought to me with a bluish cast, and many physicians were puzzled. One woman brought around a pot of urine and some physicians considered it indican; but this is not blue, and indican must be oxidized to develop the blue color. The color was due to the administration of some proprietary pill which was distributed at that time and which contained methylene blue. This urine was left standing three months and then, to my surprise, it was not putrid but was well preserved and, very strange, was colorless. On loosening the cork and shaking it the urine developed a blue color. This may be new to some of the members of the section.

Dr. William Salant, Washington, D. C.: The study of the elimination of dyes is a very good field for the pharmacologist and the medical man. I have been interested in it for some time and have examined a number of water-soluble and fat-soluble dyes with reference to their elimination. I was impressed with the fact that elimination by the liver in animals poisoned with hydrazin was the same as in normal animals. I think, however, some dyes will be found the elimination of which in the bile is different when the liver is normal and after it has undergone pathologic changes. The fact that in inflammatory conditions staining of certain organs with some dyes has been observed, whereas the same organs when normal remain unaffected by such dyes, is, I think, promising, for it may prove of value in the diagnosis of hepatic diseases.

Dr. Russell L. Cecil, New York: As to the possible toxicity of these solutions, we have injected about sixty to seventy patients intravenously and in only one did we get symptoms of toxicity, and this was, I think, because we allowed the solution to get cold. It is true that our solutions must be made up fresh with distilled water, and in using the concentrated solution it is desirable to keep it warm. This patient's temperature rose to $103 \mathrm{~F}$., with chills, but the symptoms passed away in a few hours. As to the therapeutic effect, we tried one case of ulcer of the leg with granulating edges the size of a dollar which had been treated by various methods a year or two, and by constantly injecting the dyes the patient's eyes got very red, but he said he would rather be red than have the ulcer, and we injected time and again. The ulcer improved under the treatment but the patient got restless and in five or six weeks we let him go home. As to the cosmetic effect, in the anemic patients we grt a wonderful color which will not rub off.

\section{AN APPARATUS FOR THE STUDY OF THE DISSOCIATION OF OXYHEMOGLOBIN *}

\author{
W. G. MacCALLUM, M.D. \\ NEW YORK
}

The work of Bohr, Barcroft and others has shown the importance of electrolyte contents, reaction, etc., in connection with the power of hemoglobin to absorb oxygen when exposed to this gas at different pressures. Determinations of the amount absorbed at successive low pressures of oxygen give a characteristic curve which is modified by various changes in the medium in which the hemoglobin is dissolved or suspended, and it seemed possible that the comparison of such curves, made with blood in which the nature of changes in the plasma were known, with others made from normal blood might give a clue as to the abnormality of the plasma. There are many other directions in which such determinations might be useful.

Barcroft's instrument allows a direct comparison between the amount of oxygen absorbed by a cubic centimeter of blood at a low oxygen pressure with that absorbed at the point of saturation. It appears to give no absolute or quantitative result, so that it would be difficult to follow daily changes in the blood, except by comparison with some standard.

With the idea of securing absolute rather than relative figures, the apparatus herein described was planned. It is, as the drawing shows, simply a modification of Van Slyke's apparatus for measuring the combining power of the blood for carbon dioxid. The modification consists essentially in lengthening the stem which is narrowed above and below, and graduating this stem throughout. The narrow parts, above and below the central wide tube, contain each 2 c.c., the graduation being in twentieths of a cubic centimeter. The broader part of the tube contains 48 c.c. graduated in tenths, so that the apparatus from the upper tap to the beginning of the lower narrowed portion contains 50 c.c.

A centimeter scale is fixed close to the glass apparatus, which itself is clamped in such a way as to occupy always the same position. The mercury bulb is supported in a ring which is raised or lowered by a cord that passes over a pulley to wind on a drum which can be turned by hand by the attached wheel. Close to it is fastened another centimeter scale. Since it is difficult to read the level of the mercury in the bulb against the scale, a float is arranged with a needle which plays directly on the scale and is adjusted to indicate the figure which corresponds with that read on the other scale from the level of the mercury in the glass apparatus when the taps are open to the atmospheric pressure.

By filling the apparatus with mercury, closing the upper tap, and lowering the mercury bulb, one may read off the barometric pressure by comparing the levels of mercury read from both scales. When the interior of the tube is wet, the barometric reading is lowered on account of the water vapor. The degree of lowering is practically constant with the small amount of fluid, and the wet barometric reading is used throughout, since all measurements must be made

* From the Department of Pathology, Columbia University, College of Physicians and Surgeons.

Read before the Section on Pathology and Physiology at the Sixty-Eighth Annual Session of the American Medical Association, New York, June, 1917 